\title{
NUEVAS PERSPECTIVAS DE LA TAFONOMIA EVOLUTIVA: TAFOSISTEMAS Y ASOCIACIONES CONSERVADAS
}

\author{
S. Fernández López [*]
}

\begin{abstract}
RESUMEN
Varios conceptos básicos de la Tafonomía evolutiva son tratados en este artículo. Hay una discusión general de los procesos principales que controlan la conservación tafonómica. Diversos enfoques para la aplicación de la teoría de la comunicación al registro fósil han sido revisados y sintetizados. Los problemas de resedimentación versus retrabajamiento reciben especial atención como fenómenos tafonómicos. Se hacen algunas observaciones sobre el concepto de condensación.
\end{abstract}

Palabras clave: Paleontología. Paleoecología. Tafonomía. Fosilización. Teoría evolutiva.

\section{Abstract}

Several basic concepts of the evolutionary Taphonomy are examinated in this paper. There is a general discussion of the main processes controlling fossil preservation. Approaches for the aplication of communications theory to the fossil record are reviewed and summarized. The problems of redeposition (Umlagerung) vs reworking (Aufarbeitung) receives special attention as taphonomical phenomena. Some remarks on the concept of condensation are made.

KEY wORDs: Paleontology. Paleoecology. Taphonomy. Fossilization. Evolutionary theory.

\section{Introducción}

En este artículo exponemos los conceptos de asociación conservada y de sistema tafonómico, o tafosistema, además de otros conceptos directamente relacionados con ellos y el de evolución tafonómica, con el propósito de ofrecer una visión sintética de la tafonomía evolutiva. Por este motivo nos ocuparemos de los mecanismos y procesos tafonómicos principales, más que de las diferentes clases de resultados posibles. El reconocimiento o la interpretación de procesos tafonómicos evolutivos, o de entidades conservadas de niveles de organización supraindividuales, no ha de ser necesariamente factible o deseable $y$, en algunos casos concretos, puede estar justificada la utilización de planteamientos individualistas y globalistas. Sin embargo, en el estado actual de los conocimientos, es preferible utilizar un planteamiento sistemista, por razones lógicas y metodológicas, y evolucionista, por su relevancia térica en Paleontología. Naturalmente, cualquier "-ismo" es indeseable.

\section{Acumulación de restos $y / 0$ señales producidas por entidades biológicas}

Desde hace unas décadas, varios autores han utilizado conceptos y términos de la teoría de la información para resolver problemas paleontológicos (cfr. Tasch, 1965, 1969; Beerbower \& Jordan, 1969). Paradójicamente, este enfoque, más que servir para plantear y resolver mejor problemas nuevos y antiguos, parece ser que sólo permite poner de manifiesto la ambigiuedad e imprecisión de algunos conceptos y métodos particulares de la Paleontología. Así, por ejemplo, el problema inicialmente planteado como "la transformación de los restos orgánicos componentes de la Biosfera en componentes de la Litosfera" (Efremov, 1950) ha sido convertido en el de la "transmisión de la información desdo la Biosfera hasta la Litosfera"; pero los conceptos de "registro fósil" y "asociación fósil" siguen siendo demasiado imprecisos para muchos autores; y los procesos de desenterramiento de restos $\mathrm{y} / \mathrm{o}$ señales de entidades bioló gicas históricas son incomprensiblemente tratados como procesos bioestratinómicos.

Diferentes cuestiones relacionadas con el carácter "sedimentario" de los fósiles han sido tratadas en numerosos trabajos. Por ejemplo, se ha dicho que "a geologist who calls a coral reef esedimentary» is extending the normal idea of sediments for sake of making his classification comprehensive and that is in his own interest to be aware of what he is doing. But coral reefs can also be considered as constituted of solid material which has come to rest upon the solid earth's surface by withdrawal of calcium carbonate from the sea water by biochemical processes. In

(*) Departamento de Paleontología de la Universidad Complutense de Madrid e Instituto de Geología Económica del C.S.LC. 
fact this applies to most fossils and it is a matter of opinion whether we consider them as ordinary constituents of sedimentary rocks or as objects that should be distinguished from sediments and treated separately" (Bayly, 1969, en Hoedemaeker, 1973, pág. 15). Este párrafo, a nuestro parecer, expresa bastante bien la idea generalmente aceptada por los geólogos actuales, según la cual los fósiles pueden ser considerados como partículas sedimentarias.

Respecto a esta idea, aunque los restos $\mathrm{y} / \mathrm{o}$ señales de entidades biológicas pretéritas hayan sido conservados dentro de (o en) materiales del registro estratigráfico, conviene señalar que los fósiles no pueden ser tratados sistemáticamente como partículas sedimentarias de naturaleza especial sin restringir el dominio de aplicabilidad del concepto de fósil, excluyendo la mayoría do las señales y muchos restos de entidades biológicas pretéritas. La solución pragmática adoptada por algunos autores que consideran ciertos fósiles como partículas sedimentarias no-sedimentadas sirve para evitar el problema teórico, pero no lo resuelve. El proceso de fosilización no implica que los restos y/o señales producidos por entidades biológicas pretéritas hayan sido sedimentados: la fosilización puede ser exclusivamente de información y no de materia. Para este proceso de entrada de información en la Litosfera, a partir de la Biosfera, que puede estar o no acompañada de materia y que no implica sedimentación, hemos utilizado el término acumulación propuesto por Efremov (1959, pág. 93). Es decir, cualquier elemento registrado en la actualidad ha tenido que ser acumulado dentro de, o en, materiales de la Litosfera, pero no es necesario que haya sido sedimentado después de ser producido. También cabe señalar que la acumulación de restos y/o señales puede ocurrir en materiales de origen no-sedimentario. $\mathbf{Y}$, como última salvedad, que la acumulación de elementos producidos por entidades biológicas (un proceso tafonómico al cual han estado sometidos todos los fósiles) no debe ser confundida con la concentración de elementos conservados (una propiedad actual de las asociaciones).

Volviendo a la última afirmación del párrafo mencionado, también destacaremos que para poder tratar los fósiles como objetos de naturaleza propia, lógicamente disociables de otros constituyentes de los cuerpos rocosos en que se encuentran, es condición necesaria y suficiente utilizar el concepto de conservabilidad tafonómica; lo cual no implica aceptar como presupuesto simplificador que la Paleontología sólo tiene relaciones binarias con otras Ciencias Geológicas (Fernández-López, 1982, 1984). Las entidades biológicas intervienen en los procesos sedimentarios, y el concepto de sistema biosedimentario da cuenta de este hecho (cfr. Rat, 1984), pero los fenómenos tafonómicos no son biológicos, ni sedimentológicos.

\section{Modalidades de transferencia de materia e información biológicas entre partes inestables y estables de la Litosfera}

Hay dos procesos de alteración tafonómica que pueden afectar a los restos y/o señales de entidades biológicas pretéritas después de haber sido acumulados: la resedimentación y el retrabajamiento. La resedimentación consiste en el desplazamiento sobre el substrato, antes de ser enterrados, de elementos previamente acumulados; este desplazamiento no ha de ser necesariamente lateral (por transporte sobre el substrato) y puede ocurrir, prácticamente, en el mismo lugar de acumulación. El netrabajamiento implica desenterramiento y desplazamiento, desde la posición original do enterramiento, de entidades previamente acumuladas o resedimentadas. A estos dos procesos tafonómicos sólo estarán sometidos los elementos conservados que son mecá nicamente inestables; y el límite entre las partes inestables y estables de la Litosfera puede ser utilizado para distinguir el enterramiento parcial o temporal del enterramiento total o permanente (cfr. Hanson, 1980); pero, mientras que la resedimentación de elementos conservados es un proceso bioestratinómico, el retrabajamiento de elementos es un proceso fosildiagenético. Como corolario de estas tres modalidades de transferencia de información biológica y materia taxonómicamente determinable, los distintos estados mecánicos de conservación en que se pueden encontrar los elementos conservados durante su enterramiento permanente son tres: acumulado, resedimentado y retrabajado. $Y$ cualquier elemento se encontrará en sólo uno de estos tres es-

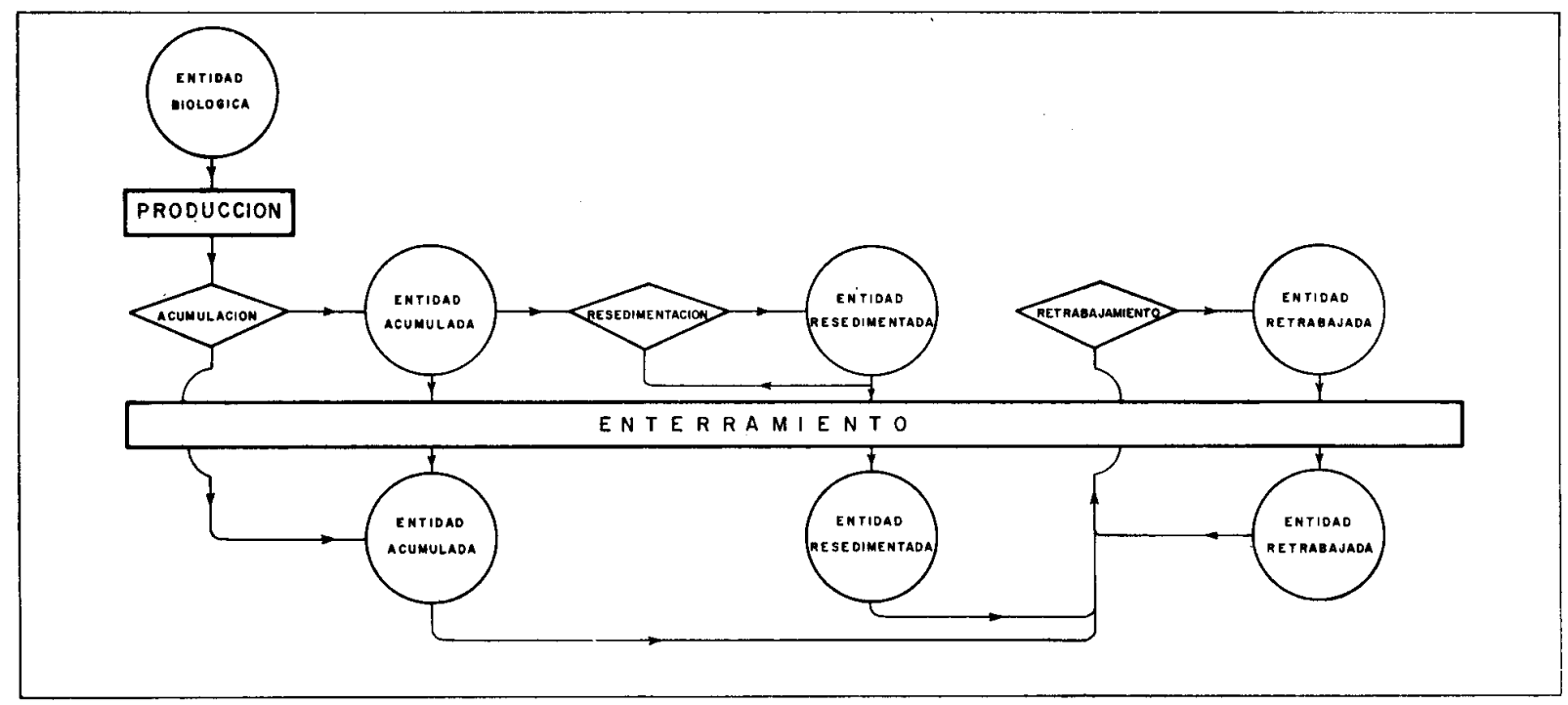

Fig. 1.-Diagrama de flujo que representa las relaciones entre las diferentes modalidades de transferencia de materia e información a unos determinados materiales de la Litosfera, a partir de una entidad biológica, y los correspondientes estados de conservación de las entidades conservadas (hayan experimentado o no transporte). 
tados posibles, con independencia de que esté completa o incompletamente conservado (fig. 1). Además, estos dos procesos de alteración tafonómica, como consecuencia de los cuales las entidades conservadas adquieren nuevas propiedades secundarias, no son necesariamente destructivos: puede ocurrir que entre los elementos producidos por una entidad biológica, los retrabajados o resedimentados tengan mayor conservabilidad que los acumulados simultáneamente con ellos.

A pesar de las diferentes repercusiones que tienen, estos dos procesos de alteración tafonómica casi nunca han sido distinguidos en los estudios tafonómicos publicados y, 10 que es peor, suelen ser denotados arbitrariamento con los términos: removilización, remoción, resedimentación y retrabajamiento. A fin de evitar esta arbitrariedad, ya hemos explicitado mediante criterios diagnósticos y ejemplos (Fernández López, 1980) el significado tafonómico de las tres clases de términos que se emplean con más frecuencia: acumulación (accumulation, Lagerung), resedimentación (redeposition, Resedimentation, Umlagerung), y retrabajamiento (reworking, Aufarbeitung). Y aunque en el caso de las conchas de ammonites las tres clases de términos no han sido caracterizadas independientemente, sí lo han sido positivamente, $y$ pueden ser utilizados para formular enunciados analíticos no-hipotéticos. Este sistema de clasificación de los elementos fósiles, que considera como posibles tres estados mecánicos de conservación indicativos de una secuencia evolutiva, posibilita una ordenación secuencial (correlacionable con una temporal del proceso de fosilización) de las diferentes clases de restos y/o señales presentes en cada asociación concreta. También permite reconocer en cualquier asociación conservada diferentes grados de remoción o removilización (es decir, resedimentación y/o retrabajamiento) en función de la proporción de elementos acumulados respecto a los resedimentados y/o retrabajados; pero la remoción denota una propiedad de las asociaciones conservadas, no de los elementos, que puede ser emergente: una asociación removilizada (contenida en un nivel de removilización o de otra clase) no ha de estar exclusivamente constituida por elementos resedimentados y/o retrabajados, sino que también puede comprender elementos acumulados.

Otros asuntos de interés paleontológico son los problemas linguísticos que plantea el término retrabajamiento. Ahora bien, a pesar de su importancia, estos temas no forman parte de los objetivos de este artículo.

\section{Asociaciones registradas y sistemas tafonómicos}

Para reconstruir procesos tafonómicos evolutivos es necesario utilizar, además de conceptos individuales y de clase, conceptos relacionales y cuantitativos; sin ellos sólo se pueden reconstruir e interpretar estados y cambios de estado.

Por otra parte, al término entidad registrada, fosilizada o conservada, no debe dársele un significado platónico: con él hacemos referencia a un elemento o a un grupo de elementos. Por ejemplo, una asociación registrada puede ser considerada como un sistema concreto, constituido por elementos interrelacionados, en el cual pueden reconocerse su composición (o conjunto de partes de la asociación), estructura (que es el conjunto de relaciones, por ejemplo espaciales, entre los miembros de la asociación, o entre miembros de la asociación y miembros del medio en que se encuentran) y medio (o conjunto de elementos que no constituyen la asociación, pero actúan sobre ella o están afectados por ella; cfr. Bunge, 1980).

Existen entidades registradas a diferentes niveles de organización. La entidad registrada más global está constituida por todos los restos y/o señales de entidades biológicas pretéritas conservados en la Litosfera; esto es lo que frecuentemente ha sido llamado "registro fósil". A niveles de organización inferiores, también constituyen entidades con- servadas los grupos de elementos funcionalmente distintos que han podido dar lugar a elementos de su misma clase taxonómicamente determinable; estos grupos tafonómicos, también llamados tafones (Fernández López, 1982), pueden haber aparecido durante el proceso de fosilización a consecuencia de la alteración tafonómica de un tafón preexistente.

Una asociación registrada es un grupo de restos $\mathrm{y} / \mathrm{o}$ señales de entidades biológicas pretéritas (producido por uno o más taxones) coincidentes en el registro estratigráfico. Además, se asume que la coincidencia en el registro estratigráfico implica interacciones entre los constituyentes individuales, bien directamente o bien por la influencia que los elementos entre sí ejercen sobre el ambiente común (cfr. Olson, 1980, pág. 10). Los elementos de cualquier asociación registrada están contenidos en uno o más niveles estratigráficos. Si cada asociación registrada es representable por su composición, estructura y medio, cualquier cambio de asociación podrá ser caracterizado como un cambio de alguna do sus propiedades. Por ejemplo, la estructura integrada de interacciones entre los elementos del sistema tafonómico permite definir los límites del sistema tanto cspacial como temporalmente; si la estructura es alterada, la entidad conservada (en este caso la asociación) deja de existir (por destrucción o por transformación en otra entidad conservada).

\section{Tafosistemas y energía externa}

Cualquier entidad biológica tiene $n$ "bits", u otras unidades de información codificada, que comprenden desde propiedades morfológicas, estructurales o químicas, hasta ecológicas y evolutivas. La suma de estas unidades de información $(S h)$ podemos considerar que es el mensaje susceptible de ser transmitido desde la biosfera hasta la litosfera. Tras la producción y fosilización de la información, un lapso temporal de millones de años puede haber transcurrido desde que existió la entidad biológica en cuestión hasta que se procede a la obtención o descodificación de la información registrada (Tasch, 1965, 1969). Los procesos de producción y fosilización, además del desenterramiento (que generalmente constituye un bucle de retrorregulación diferida entre procesos bioestratinómicos y fosildiagenéticos, pero que en ocasiones también afecta a las entidades biológicas) determinan la información y materia recuperables (fig. 2). A través de estos tres procesos fundamentales, la información biológica histórica habrá sido modificada por: redundancia $(R)$, transformación $(T)$ y/o pérdida irrecuperable de materia e información $(D)$

La redundancia $(R)$ es la repetición del mismo mensaje de una entidad biológica $(R p)$ o de una entidad producida (Rf) (cfr. Fernández López, 1982, pág. 249). La producción de elementos múltiples por una entidad biológica, la producción secundaria de elementos durante la fosilización, la formación de impresiones externas e internas, moldes, etc., ejemplifican diferentes procesos de redundancia en el registro original, Los efectos de la redundancia deben ser eliminados de la información registrada antes de intentar resolver el "ruido" o la transformación de la información original. El grado de contenido informativo de una entidad registrada puede ser expresado mediante el porcentaje de redundancia.

La transformación o "ruido" (T) de la información biológica durante la producción $(p)$ y la fosilización $(f)$ ocurre por distorsión y/o reacción (en composición, estructura y medio) de las unidades de información biológica. La interferencia entre sucesivas señales y/o restos producidos, su disolución, desgaste, fragmentación, deformación mecánica (por compactación diagenética gravitacional o por deformación tectónica) son algunos de los "items" que pueden ser considerados como "ruido" en la información registrada (cfr. Tasch, 1969). Además, la ganancia de información y materia a partir de otros sistemas biológicos, coexistentes 
$(G p)$ o no $(G f)$, también contribuye a transformar la información conservada de cualquier entidad biológica histórica singular; dichas ganancias implican la pérdida en otras áreas de elementos conservados y su desplazamiento (por resedimentación, retrabajamiento, infiltración, contaminación, etc.) e introducción en otra entidad conservada que se encuentra en la fase bioestratinómica (b) o fosildiagenética $(g)$. La proporción entre información original $(S h)$ e información transformada (ST) es un criterio para estimar las relaciones servada; el proceso de exportación de información, al igual que el de importación o ganancia, puede incrementar la cantidad de información del tafosistema.

La energía que da lugar a la formación de cuerpos rocosos también contribuye a la conservación y organización de los sistemas tafonómicos; a dicha energía se le puede calificar de externa. Al menos en los tafosistemas subacuáticos, uno de los mejores predictores de la conservabilidad es la disponibilidad en cada lugar concreto de energía ex-

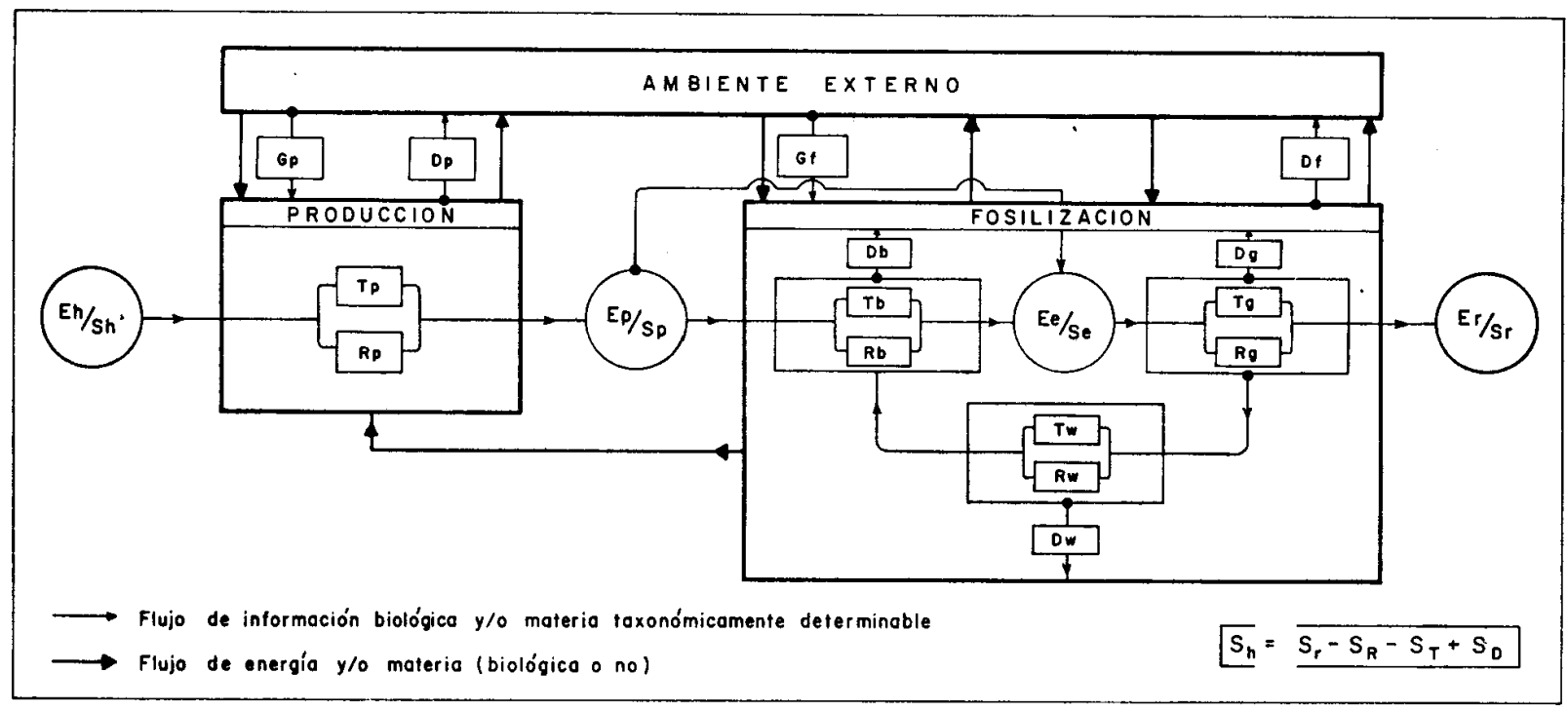

Fig. 2.-Diagrama de flujo de información, materia y/o energía entre un sistema tafonómicopaleoceológico y su ambiente externo. La relación algorítmica entre la información biológica (Sh) y la información registrada (Sr) está expresada en función de la información redundada [SR, durante la fosilización (f) y la producción (p)], la información transformada (ST) y la información perdida (SD). Los procesos bioestratinómicos (b) y/o fosildiagenéticos (g) integran los procesos tafonómicos (f). Las entidades producidas (Ep) dentro de materiales de la Litosfera son entidades enterradas (Ee) que no han experimentado procesos bioestratinómicos. Los procesos de retrabajamiento (w) y/o resedimentación regulan y modifican la información enterrada (Se); además, pueden afectar la producción de las entidades biológicas (Eh). Las entidades registradas (Er) también pueden haber estado controladas (directamente o a través del ambiente externo) por la energía y/o materia perdida durante la producción y la fosilización. La pérdida de materia e información (D) en el sistema tafonómico-paleoecológico, comprende las pérdidas durante los procesos de producción (Dp) y fosilización (D), así como la materia e información no-producida. La ganancia de información biológica $\mathrm{y} / \mathrm{o}$ materia taxonómicamente determinable $(G)$ a partir de otras entidades biológicas, coexistentes (Gp) o no (G), contribuye a modificar la información conservada de cualquier entidad biológica singular. Cualquier entidad biológica (Eh) tiene $n$ unidades de información (siendo $n$ un número finito, concreto o no), pero la información biológica (Sh) no tiene existencia propia.

entre la entidad biológica y los agentes transformadores de la información producida. La información transformada ha de ser interpretada antes de intentar elucidar la información perdida.

Los efectos de la llamada pérdida irrecuperable de materia $e$ información $(D)$ incluyen desde la información no-producida hasta la perdida durante los procesos de producción $(D p)$ y fosilización $(D f)$; pero, al menos durante la fosilización, consisten en perder materia más que información; en cualquier caso, la información registrada permite averiguar la materia organizada perdida en un sistema tafonómico, y que ha dejado de existir en su estado taxonómicamente determinable (fig. 2). La pérdida de información, o mejor dicho, la información exportada al ambiente externo, no implica una reducción en la cantidad de información con- terna (cfr. Margalef, 1981). Esta energía es usada durante la producción de elementos, transporte, desarticulación, fragmentación, aspiración de partículas sedimentarias, resedimentación, formación y mantenimiento de sedimentos, retrabajamiento, mineralización, etc. Es decir, la producción original y secundaria de elementos conservados y la alteración tafonómica están reguladas por la disponibilidad de energía externa. Cuando es escasa dicha energía, entonces el sistema tiende a la destrucción y a su desaparición. De acuerdo con lo expuesto en párrafos anteriores, el ambiente externo puede ser entendido como aquellos otros elementos (o sistemas) que están afectados (pero no controlados) por la energía, materia o información exportadas desde el tafosistema en cuestion. 


\section{Asociaciones mezcladas}

Los elementos que constituyen una asociación registrada pueden ser autóctonos o alóctonos respecto al lugar en que han sido producidos. En contra de lo que frecuentemente ha sido escrito, el término alóctono no debería ser utilizado como sinónimo de exótico; mientras que el primer término hace referencia a la distancia recorrida por los elementos desde el lugar en que han sido producidos hasta el sitio dondo están conservados, el segundo término destaca la diferente naturaleza de los elementos de una entidad biologica supraindividual. Análogamente, tampoco son sinónimos los términos autóctono e indígena, aunque muchos autores no hayan respetado estas diferencias conceptuales (cfr. Spatella et al., 1983, Turner, 1982; Hoedemaeker, 1973). Es decir, los términos indígena y exótico hacen referencia a propiedades de entidades biológicas, mientras que autóctono y alóctono refienen propiedades de entidades conservadas. La autoctonía en sentido estricto consiste en la coincidencia entre los lugares correspondientes a la entidad producida y a la entidad conservada. Tampoco deben confundirse estos conceptos con el estado en que han sido encontradas las entidades obtenidas (in situ - ex situ o "rodado").

Los elementos constituyentes de una asociación registrada habrán sido producidos simultánea o heterócronamente entre sí y/o respecto a la roca que los contiene. Las relaciones espacio-temporales entre los elementos conservados son independientes del estado mecánico de conservación de dichos elementos. Así, por ejemplo, el carácter acumulado de todos los elementos de una asociación no prueba su origen espaciotemporal común. Todos los elementos acumulados que se conservan en la posición en que han sido producidos son autóctonos; este criterio de "posición en que han sido producidos", en vez del criterio de "posición de vida", es más apropiado para los restos de entidades biológicas y, además, es aplicable a las señales de actividad biológica. La resedimentación y/o retrabajamiento de elementos no implica desplazamiento lateral y, por tanto, tampoco aloctonía, además, ya que estos dos procesos pueden afectar simultáneamente a un conjunto de elementos, tampoco implican heterocronía entre los elementos que constituyen una asociación resedimentada o retrabajada. Algunas propiedades de las asociaciones de elementos conservados confirman su carácter autóctono, al excluir que hayan sido transportados sin selección; por ejemplo, la coexistencia de elementos de los diferentes estadios de desarrollo ontogénico en el seno de una población monoespecífica (Hallam, 1967; Gall, 1976, pág. 107). También las propiedades de los materiales formados durante la producción y acumulación de los elementos conservados en ellos permiten refutar la autoctonía, cuando al ambiente de formación de la roca es incompatible con el de producción y/o acumulación de elementos o de entidades supraindividuales.

Numerosos autores han distinguido las asociaciones que contienen elementos alóctonos (tafocenosis, Quenstedt, 1927) de las asociaciones de elementos autóctonamente conservados, designando a estas últimas con los nombres de tanatocenosis autóctonas (Wasmund, 1926, si se trata de restos) e ichnocenosis (Davitashvili, 1945, para referirse a señales). Para los productos de ciclos vitales, tales como exuvias, hojas y esporomorfos, ha sido propuesto el término pseudocenosis (Martinson, 1954). Aquellas asociaciones que sólo tienen algunos elementos alóctonos han sido mencionadas en la bibliografía con los términos de tanatocenosis alóctona y tafocenosis. Otros autores, sin embargo, han utilizado los términos tafocenosis y tanatocenosis para distinguir diferentes tipos de asociaciones en la secuencia temporal de eventos que han afectado a cualquier conjunto de restos orgánicos, discriminando entre tanatocenosis (restos de organismos que murieron juntos), tafocenosis (restos de organismos que fueron enterrados juntos) y orictocenosis (fósiles que están, o que han sido encontrados, juntos) sin tener en cuenta el carácter autóctono o alóctono de sus elementos (cfr. Efremov, 1950, pág. 108; Hecker, 1965; Lawrence, 1968; Müller, 1979). Ante esta diversa aplicación de los conceptos, durante los años setenta, diferentes autores han intentado definir de la mejor manera posible estos términos; con lo cual han evitado que siguieran siendo arbitrariamente aplicados, pero no se ha eliminado su defecto fundamental: designan conceptos individuales y globalistas (cfr. Ghiselin, 1969, página 72).

Una asociación conservada o registrada es una asociación mezclada si sus elementos constituyentes corresponden a dos o más entidades biológicas de ambientes diferentes; dichas diferencias ambientales pueden ser exclusivamente espaciotemporales. Por tanto, casi todas las asociaciones registradas serán asociaciones mezcladas y, en consecuencia, lo importante en cada asociación concreta no es saber si es de esta clase, sino averiguar el grado de las diferencias ambientales correspondientes a sus elementos. Entendido así, el concepto de asociación conservada (mezclada o no) es lógicamente más fuerte que el de tanatocenosis o tafocenosis.

Al tratar cuestiones referentes a procesos de necrocinesis (Quenstedt, 1972; es decir, los desplazamientos ascendentesdescendentes y/o laterales de las entidades producidas, experimentados antes de ser enterradas y que generalmente se llevan a cabo por rodamiento, saltación y/o suspensión) es conveniente tener en cuenta que las evidencias de deriva necroplanctónica implican que ha habido necrocinesis pero, por las mismas razones que la afirmación conversa no es cierta, las evidencias de resedimentación no implican que hayan ocurrido procesos de transporte; además, los procesos de necrocinesis pueden ocurrir antes de que los elementos producidos lleguen a estar acumulados (cfr. Tintant, 1984). En cualquier caso, el grado de alteración tafonómica alcanzado durante la fase bioestratinómica por una entidad conservada es proporcional a la intensidad y duración de dicho proceso, con independencia de que la entidad haya o no experimentado desplazamientos laterales; más concretamente, las evidencias de colonización por "epizoarios" durante la fase bioestratinómica de una entidad conservada no implican que dicha entidad ha experimentado deriva necroplanctónica o transporte.

El desplazamiento de elementos conservados en, o dentro de, los materiales de la Litosfera, se debe a la acción de agentes físico-químicos o biológicos. Por ejemplo, los organismos necrófagos o carroñeros remueven los restos y/o señales orgánicas de la interfase deposicional; los endobiontes también suelen dar lugar a mezclas de elementos en la parte superficial del substrato (Fürsich, 1978; Matissof, 1982). En ocasiones, la mezcla de elementos conservados también ha ocurrido por causas tectónicas (fracturación, diapirismo, etcétera). En resumen, el proceso de mezcla entre los constituyentes de una entidad registrada puede haber ocurrido durante las fases bioestratinómicas $\mathrm{y}$ fosildiagenéticas del proceso de fosilización y, en principio, convendría distinguir en cada caso concreto si se trata de una mezcla bioestratinómica o fasildiagenética. Otros efectos de mezcla resultan por el método de muestreo utilizado para estimar la entidad registrada, pero no trataremos el tema en este artículo.

Si es importante destacar que la causa de mezcla, o los factores que determinan la mezcla, no siempre es externa a las entidades mezcladas: algunos elementos conservados inducen el proceso de mezcla y determinan la composición y la estructura de las futuras asociaciones producidas y conservadas (fig. 2); así, por ejemplo, la concentración de restos orgánicos sobre el fondo marino pueden inducir cambios en la comunidad bentónica al proporcionar los elementos conservados un substrato para la epifauna incrustante o fijada mediante biso, o para los organismos perforantes (cf. Fürsich, 1978, 1979). También puede ocurrir que la materia resultante de la descomposición de restos orgánicos sólo sea letal para algunos organismos. A este respecto, al menos en el caso de algunas entidades biológicas, es útil distinguir si la producción original de elementos ha sido autogénica (cau- 
sada por la intervención de fuerzas internas, según Müller, 1979, pág. 6) o, por el contrario, alogénica (debido a la actuación de fuerzas externas, Müller, 1979; o en función de la energía externa utilizada en favorecer la producción); otras concepciones más simplistas del proceso de producción utilizan los términos voluntaria/involuntaria o endó gena/exógena. En cualquier caso, la producción de restos y/o señales no debe ser confundida con la productividad orgánica: son dos procesos diferentes que no han de estar necesariamente correlacionados.

Las condiciones de producción también pueden ser inferidas, al menos en parte, teniendo en cuenta las propiedades secundarias de la entidad registrada. La interpretación de la entidad biológica histórica a partir de la correspondiente entidad registrada, será más precisa si conocemos, en cierto grado, las condiciones de producción y la entidad histórica; pero, ya que es esta última el motivo principal de la investigación tafonómico-paleoecológica, pueden utilizarse algunas propiedades conocidas de la entidad biológica histórica y de las condiciones de producción para estimar con mayor precisión y exactitud la naturaleza de la entidad histórica en cuestión. Es decir, a partir de un número limitado de propiedades conocidas sobre la entidad biológica y sobre las condiciones de producción y fosilización, podemos inferir nuevas propiedades respecto a la entidad biológica (cfr. Shipmann, 1981). Ahora bien, las entidades biológicas históricas de diferentes niveles de organización tienen que ser independientemente interpretadas; pues, al ser de naturaleza distinta, cabe esperar que se hayan comportado diferencialmente durante los procesos de producción y fosilización. Además, si en el análisis tafonómico no se sobrepasa e nivel individual, las interpretaciones de las entidades biológicas serán necesariamente individualistas y, en consecuencia, cualquier referencia a sus propiedades emergentes no será más que una hipótesis auxiliar. Por lo tanto, al margen de que sean investigados procesos evolutivos o no, es necesario obtener evidencias a los correspondientes niveles de organización, a partir de las entidades registradas, para que las interpretaciones de las entidades biológicas históricas puedan ser fundadas y contrastables.

\section{Asociaciones condensadas}

El concepto de condensación es de gran importancia en Paleontología y ha sido ampliamente utilizado por numerosos autores, aunque con significados muy diferentes. A continuación trataremos tanto el significado del concepto como el método para poner de manifiesto el proceso o estado referido por dicho término. Estos dos aspectos, el conceptual y el metodológico, no siempre han sido distinguidos en la bibliografía que hemos consultado, y, a veces, incluso han sido confundidos como un único problema.

Según el concepto original, la condensación estratigráfica es el resultado de una sedimentación extremadamente lenta o nula que se mantuvo durante un largo período de tiempo; y las arcillas rojas que se encuentran actualmente en las regiones abisales son un ejemplo de depósitos condensados (cfr. Heim, 1934, 1946; Rod, 1946; Schaub, 1948). Aceptada esta idea, dicho resultado estará materializado por un cuerpo rocoso de potencia menor que la de otro cuerpo rocoso de referencia, o bien por una superficie sobre la que prácticamente no hubo sedimentación. Es decir, el carácter condensado de un cuerpo rocoso no es una propiedad absoluta de él, sino relativa respecto a otro cuerpo rocoso que ha sido formado durante el mismo intervalo temporal o una duración equivalente. Además, para que se lleve a cabo la condensación estratigráfica, no es necesaria la conservación de restos y/o señales de entidades biológicas: un cuerpo rocoso "azoico", a una sucesión de cuerpos rocosos, puede tener carácter condensado respecto a otro cuerpo rocoso, u otra secuencia, si el resultado de una sedimentación más escasa o nula, aunque no sea fosilífero (Geyer y Hinkelbein, 1971, pág. 409). No obstante, la mayoria de los autores han utilizado como criterio empírico para precisar el concepto de condensación estratigráfica que los materiales condensados contienen fósiles de diferente edad (cfr. Mensink, 1960; Wendt, 1970, 1973; Seilacher, 1970, 1973, 1982; Fürsich, 1971, 1978, 1979, 1982; Gatrall et al., 1971; Geyer et al., 1971, 1973, 1974; Jenkins, 1971; Krystyn et al., 1971; Dietl, 1974; Seifried, 1981; Gebhard, 1982; Hagdorn, 1982). Esta regla de decisión ha sido el motivo de que la condensación estratigráfica y la llamada "condensación faunística" hayan sido tratadas como el mismo problema; cuando, en realidad, los dos procesos son independientes: se conocen asociaciones fósiles condensadas en materiales que son el resultado de una sedimentación abundante, rápida y constante, al igual que existen asociaciones no-condensadas en materiales condensados. Los resultados de los procesos de condensación tafonómica y de condensación estratigráfica no son necesariamente coincidentes en los mismos niveles estratigráficos. $Y$ las únicas asociaciones condensadas que pueden ser utilizadas como criterio para confirmar la condensación estratigráfica en un cuerpo rocoso son aquellas cuyos elementos han sido producidos sucesivamente entre sí y penecontemporáneamente respecto a las rocas que los contienen; por este motivo, algunos autores han destacado que los fósiles retrabajados no pueden ser utilizados para reconocer niveles u horizontes de condensación.

Una asociación registrada es una asociación condensada si sus elementos constituyentes corresponden a dos o más entidades biológicas temporalmente sucesivas. $\mathrm{Y}$ la condensación tafónómica es el proceso de mezcla de restos y/o señales de entidades biológicas históricamente sucesivas. La condensación tafonómica o la llamada "condensación faunística" es, pues, una propiedad supraindividual; pero el término condensación faunística debería ser utilizado, exclusivamente, para expresar relaciones paleobiogeográficas entre dos o más entidades biológicas históricas. La existencia de una entidad condensada (por ejemplo, una asociación conservada, una población tafónica, etc.) en un nivel estratigráfico no significa que los elementos constituyentes hayan sido enterrados en fases sucesivas, sino que las entidades biológicas que los han producido no fueron coexistentes. La mezcla de elementos que constituyen una asociación condensada puedo haber ocurrido antes o después del enterramiento de la asociación; en el primer caso, podría hablarse de condensación bioestratinómica, y, en el segundo, de condensación fosildiagenética. En uno y otro caso, la mezcla de elementos que integran una asociación condensada puede haber sido simultánea o sucesiva entre sí y anterior, penecontemporánea o posterior a la formación del cuerpo rocoso en que están conservados.

Una asociación conservada puede estar condensada aún cuando todos sus elementos presentan el mismo estado mecánico de conservación. Este es el caso de aquellos elementos que han resultado de varias acumulaciones sucesivas en los mismos materiales (por ejemplo, varias generaciones de endobiontes). $Y$, al igual que el carácter acumulado de los elementos no excluye que haya habido condensación. tampoco el carácter retrabajado garantiza que un conjunto de elementos corresponda a dos o más entidades biológicas históricamente singulares. Sin embargo, cuando en una asociación registrada hay elementos con diferentes estados de conservación y, más concretamente, junto a elementos retrabajados hay otros acumulados o resedimentados, las diferencias de estado de conservación permiten una ordenación secuencial de al menos dos clases de restos y/o señales (acumulados $v s$. retrabajados) que es correlacionable con el orden temporal de fosilización.

Por lo tanto, para llegar a establecer que una asociación de fósiles es una asociación condensada sólo es necesario tener en cuenta las relaciones topológicas que, basadas en los caracteres secundarios resultantes de la alteración tafo- 
nómica, existen entre los elementos conservados. Al probar que las transformaciones tafonomicas experimentadas por dos o más elementos, o que la evolución tafonómica de diferentes tafones, han tenido distintas duraciones geológicas, se prueba que en una misma asociación registrada están condensados los restos y/o señales de sucesivas entidades biológicas; ya que, conociendo las sucesivas transformaciones tafonómicas experimentadas por dos o más elementos registrados y las relaciones entre ellas, pueden ser elucidadas las relaciones de coexistencia-sucesión entre los elementos durante los procesos de producción y mezcla, y las relaciones temporales entre sus correspondientes entidades productoras. Pero es contrario a la teoría bioestratigráfica vigente utilizar argumentos biocronológicos apriorísticos, basados en relaciones temporales que han sido interpretadas con los fósiles de otros organismos taxon6micamente semejantes y que han sido encontrados en otras áreas geográficas (cfr. Fernández López, 1980).

\section{Otras perspectivas de la conservabilidad tafonómica}

Las entidades registradas (elementos, tafones, asociaciones, tafosistemas, ...) son los resultados observables (directa 0 indirectamente) del proceso de alteración tafonómica que ha actuado sobre entidades previamente conservadas. La entidad conservada no ha de ser necesariamente una entidad morfológica, puede ser un cambio de tasa en un proceso físico-químico o un cambio de orientación en el espacio (por ejemplo, un cambio de la llamada "biotextura"). Y no debe ser confundido el sedimento, las partículas sedimentarias o un cuerpo rocoso (que actúan como transmisore de información) con las propiedades ambientales del tafosistema (que intervienen como agentes alternativos); ađemás la alteración tafonómica, entendida o no como un proceso de retención selectiva, no es necesariamente destructiva, aun cuando pueda modificar la información originalmente pro ducida.

Por otra parte, también es importante destacar que las entidades conservadas requieren tiempo para materializarse. La distinción entre una respuesta conservativa y una respuesta ordinaria puede hacerse en base a criterios temporales. Shapere (1974) ha expuesto la validez de estos criterios en el caso de las respuestas adaptativas.

Un presupuesto de la tafonomía evolutiva es que las entidades conservadas están en equilibrio dinámico con su ambiente externo, que ellas son "sistemas dinámicos abiertos" con intercambios continuos; es decir, son sistemas "negentrópicos". Una entidad conservada no sólo está determinada por sus propiedades originales, sino también por su ambiente. Las variaciones físico-químicas entre entidades conservadas podrán estar biologicamente determinadas o ser inducidas ambientalmente, pero el conjunto de caracteres originales establece los límites para el efecto ambiental mantenimiento del "equilibrio dinámico" o estado interno relativamente estable no es un logro exclusivo de los mecanismos fisiológicos; también se consigue mediante respuestas adecuadas del comportamiento. Esto es lo que constituye la durabilidad, la capacidad de durar en un ambiente que cambia. Cualquier entidad conservada mantiene un pequeño rango de sus propiedades, incluso en ambientes ampliamente variables $y$. aunque algunos parámetros estarán más regulados que otros, la variación interna tendrá unos límites más allá de losi cuales la durabilidad será nula (Fernández López, 1982). Una entidad producida se conservará de manera diferente según los diversos componentes físicos, químicos y biológicos del ambiente a que esté sometida, y diferentes partes de una misma entidad podrán tener distintas capacidades conservativas. Algunos elementos conservados se conforman en su ambiente con respecto a diversos factores físico-químicos concretos (como, por ejemplo, la temperatura o la concentración de algunos elementos o compuestos químicos) y se regulan respecto a otros. La durabilidad representa el conjunto de mecanismos compensatorios, y las relaciones entre ellos, por el cual una entidad conservada mantiene alguna independencia respecto al ambiente externo en que se encuentra (la analogía con servosistemas de la teoría de la información puede ser útil para la Tafonomía, cfr. Proser, 1964). Los procesos de conservación tafonómica tienen límites menos amplios que el rango de ambientes físicos (reales) de la tierra; y cuanto más especializado sea un tafón, en el sentido de independencia del ambiente, mayor será el rango ambiental que podrá tolerar. Los restos y/o señales conservados serán conformes a los parámetros del ambiento externo y podrán presentar modificaciones compensatorias diferentes si son afectados por variaciones ambientales distintas. Dos grupos de elementos simultáneamente conservados sólo constituyen tafones dis-

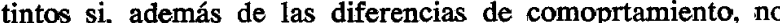
es posible entre ellos el flujo de unidades de información codificada, aun en el caso de que sean espacialmente coincidentes.

La conservabilidad de una entidad $X_{1}$ expuesta al ambiente $a_{1}$ también puede ser comparada con la de una entidad $X$ sometida al ambiente $a_{2}$. El ambiente $a_{2}$ tendrá unas propiedades cuantificables $A a, A b, \ldots A n$ que diferirán de las del ambiente $a_{t}$ por incrementos $\triangle A a, \Delta A b, \ldots \Delta A n$, cuyos valores son estimables. $Y$ cada incremento puede ser considerado como un agente alterativo (destructivo o no). Además, cualquier agente alterativo podrá ser caracterizado por su intensidad, duración, frecuencia y variabilidad; pero no ha de ser necesariamente reconocido como una entidad físicoquímica, sino que puede corresponder a alguna diferencia de energía o concentración entre los ambientes $a_{1}$ y $a_{2 *} Y$, si la entidad $X_{2}$ modifica sus propiedades respecto a $X_{1}$, cada modificación será un incremento de algún resultado $E a$ $E b, \ldots E n$. Este esquema es un instrumento para ordenar concepos sobre cualquier modificación de respuestas de un sistema (cfr. Adolph, 1964). La entidad y el ambiente utilizados como referentes podrán ser históricos o actuales (naturales o artificiales); pero este procedimiento no debe ser considerado como un enfoque alternativo al de la tafonomía evolutiva, sino como un instrumento para plantear y resolver los problemas tafonómico-paleoecológicos, ya que las conclusiones obtenidas por este procedimiento deben estar basadas en, y ser contrastadas con, evidencias positivas para cada entidad y ambiente históricos concretos (cfr. Behrensmeyer y Hill, 1980; Tasch, 1982). Además, las diferencias entre dos entidades registradas no han de ser debidas exclusivamente a factores alterativos, productivos o históricos, también pueden ser el resultado de distintas interacciones retroactivas entre las correspondientes entidades conservadas y el ambiente externo.

Al comparar varias entidades registradas, con demasiada frecuencia se encuentran diferencias fácilmente explicables en términos ecológicos a evolutivos; pocas veces se ha tenido en cuenta si esas diferencias son debidas, al menos en parte, a procesos de evolución tafonómica; y, por el contrario, las inferencias sobre las entidades biológicas históricas, las relaciones entre ellas o con sus respectivos ambientes, suelen llevarse a cabo ignorando el requisito metodológico de considerar otras hipótesis tafonómicas que den cuenta de las evidencias obtenidas en el registro fósil. Es ilógico que algunos autores admitan los modelos basados en entidades biologicas actuales con validez decreciente para entidades biologicas históricas más antiguas, pero ignoren de manera sistemática los posibles efectos de la evolución tafonómica que, por razones análogas, deberían ser considerados como más probables. Antes de interpretar las pro piedades de las entidades biológicas históricas, es necesario conocer las modificaciones tafonómicas experimentadas por sus correspondientes entidades registradas (cfr. De Renzi, 1981). 


\section{Sumario}

Considerar los fósiles como constituyentes lógicamente disociables del registro estratigráfico, no es una cuestión de opinión; para ello, se requieren conceptos que permitan disociarlos. Y si los fósiles son tratados sistemáticamente como partículas sedimentarias, entonces se restringe el dominio de aplicabilidad del concepto de fósil. La Paleontología está interrelacionada con la Biología, Estratigrafía y Sedimentología; pero los fenómenos tafonómicos no son biológicos, ni sedimentológicos.

El estudio del proceso de desenterramiento de elementos conservados pone de manifiesto la necesidad de distinguir diferentes niveles de organización para poder establecer los límites entre procesos fosildiagenéticos, bioestratinómicos y paleobiológicos. Por razones metodológicas, también es necesario discernir entre estado y acontecimiento (evento, cambio de estado) o proceso, cuando se utilizan los conceptos de resedimentación $\mathrm{y} / \mathrm{o}$ retrabajamiento. Un orden temporal del proceso de fosilización y de las correspondientes entidades registradas puede ser inferido, sin recurrir a criterios biocronológicos apriorísticos, si se tiene en cuenta el estado de conservación de los elementos constituyentes de una asociación mezclada. Las únicas asociaciones condensadas que pueden ser utilizadas para confirmar la condensación estratigráfica, en un nivel $u$ horizonte concreto, son aquellas cuyos elementos han sido producidos sucesivamente entre sí y penecontemporáneamente respecto a las rocas que los contienen. El desenterramiento puede ser considerado como un buche de retrorregulación diferida entre los procesos bioestratinómicos y los fosildiagenéticos. La alteración tafonómica, entendida o no como un proceso de retención selectiva, no es necesariamente destructiva, aun cuando pueda modificar la información originalmente producida.

Los conceptos de tafosistema y asociación conservada o registrada, junto con los de tafón y elemento, amplían la jerarquía tafonómica a diferentes niveles de organización. Estos conceptos son compatibles con la acepción actual de "registro fósil" y posibilitan la interpretación y contrastación de entidades biológicas supraindividuales. Las interpretaciones de los sistemas bio-sedimentarios del pasado pueden estar basadas en, y ser independientemente contrastadas con, los datos tafonómicos. Las entidades producidas y conservadas, si se comportan como sistemas "negentrópicos", pueden ser interpretadas, al menos en parte, descodificando o "transcodificando" la información registrada. En última instancia, este planteamiento permite discriminar, con evidencias positivas, si la información registrada es representativa o no de la información biológica histórica.

\section{Bibliografía}

Adolph, E. F.

1964. Perspectives of adaptation: some general properties. En: Dill, D. B. (Ed.): Adaptation to the Environment, 27-35 (Amer. Physiol. Soc.), Washington.

BeERBower, J. R. y JoRdán, D.

1969. Application of information theory to paleontologic problems: taxonomic diversity. J. Paleontology, 43, 1184-1198.

Behrensmeyer, A. K. e Hill, A. P. (Ed.)

1990. Fossils in the Making. Vertebrate Taphonomy and Paleoecology, 338 págs. (Univ. Chicago Press), Chicago-London.

BUNGe, M.

1980. Epistemología, 275 págs. (Ariel), Barcelona.

DE RENZI, M.

1981. Some philosophical questions about Paleontology and their practical consequences. Acta Geológica Hispánica, 16 (Concept and Method in Paleontology, Ed. J. Martinel.L), 7-23.

DIETL, G.

1974. Zur Stratigraphie und Ammonitenfauna des Dogger insbesondere des Oberbajocium des westlichen Keltiberischen Ketten (Spanien). Stuttgarter Beitr. Naturk. (B), 14, 1-21.

EFREMOV, I. A.

1950. (Taphonomie et annales géologiques). Trav. Inst. Paleont. Acad. Sci. URSS, 24, 177 págs. (trad. Ann. Centre d'etud. doc. Paleont., 4, París).

Fernández-López, S.

1980. Estudio bioestratigráfico (Ammonoidera) de materiales aalenienses en Ribarroja (Valencia). II Col. Estrat. Paleogeogr. Jurásico España (1979), Granada. Estudios geol., 35 (1979), 415-423; Cuad. Geol., 10 (1979), 55-65.

1982. La evolución tafonómica (un planteamiento neodarwinista). Bol. R. Soc. Española Hist. Nat., 79, 243-254.

1984. Enmienda para un modelo algebraico de la Geología. Bol. R. Soc. Española Hist. Nat., 40 (en prensa).

Fürsich, F.

1971. Hartgrïnde und Kondensation im Dogger von Calvados. N. Jb. Geol. Paläont. Abh., 138, 313-342.

1978. The influence of faunal condensation and mixing on the preservation of fossil benthic communities. Lethaia, 11, 243-250.

1979. Genesis, environments, and ecology of Jurassic hardgrounds. N. Jb. Geol. Paläont. Abh., 158, 1-63.

1982. Rhythmic Bedding and Shell Bed Formation in the Upper Jurassic of East Greenland. En: EINSELE, E. y SeIlacher, A. (Eds.). Cyclic and Event Stratification, 208-222 (Springer). Berlin-Heildelberg-New York.

GaLL, J. C.

1976. Environnements sédimentaires anciens et milieux de vie. Introduction à la paléoécologie, 228 págs. (Doin), París.

Gatrall, M.; Jenkyns, H. C. y Parsons, C. F.

1971. Limonitic concretions from the European Jurassic, with particular reference to the "snuff-boxes" of southern England. Sedimentology, 18, 79-103. 
Gebhard, G.

1982. Glauconitic Condensation Through High-Energy Events in the Albian Near Clars (Escragnolles, Var, SE-France). En: EINSELE, G. y SeILACHER, A. (Eds.). Cyclic and Event Stratification, 286298 (Springer), Berlin-Heildelberg-New York.

GEYER, O. F.

1973. Grundzüge der Stratigraphie und Fazieskunde. 1: Paläontologische Grundlagen. I: Das geologische Profil. Stratigraphie und Geochronologie, 279 (E. Schweizerbart), Stuttgart.

Geyer, O. F. y Hinkelbein, K.

1971. Eisenoolithische Kondensations-Horizonte im Lias der Sierra de Espuña (Provinz Murcia, Spanien) N. Jb. Geol. Paläont. Mh., 1971, 398-414.

1974. Die Grenzoolithe im Jura von Ostspanien. $N$. Jb. Geol. Paläont. Abh., 145, 17-57.

Ghiselin, M. T.

1969. (The Triunmph of the Darwinian Method), 278 págs. (trad. Ed. Cátedra, 1984, Madrid).

\section{HAGDORN, H}

1982. The "Bank der kleinen Terebrateln" (Upper Muschelkalk, Triassic) near Schwäbisch Rall (SWGermany) - a Tempestite Condensation Horizon. En: Einsele, G. y SeIlacher, A. (Eds.). Cyclic and Event Stratification, 263-285 (Springer), Berlin-Heidelberg-New York.

Hallam, A.

1967. The interpretation of size-frecuency distributions in molluscan death assemblages. Paleontology, $10,25-42$.

Hanson, C. B.

1980. Fluvial Taphonomic Processes: Models and Experiments. En: Behrensmeyer, A. K. y Hill, A. P. (Eds.). Fossils in the Making. Vertebrate Taphonomy and Paleoecology, 156-181 (Univ. Chicago Press), Chicago-London.

Hecker, R. F.

1957. (Introduction to Paleaecology), 166 págs. (trad. Elsevier, Ed. 1965, New York).

HeIM, A.

1934. Stratigraphische Kondensation. Eclog. geol. Helv., 27, 372-383.

1946. Problemas de erosión submarina y sedimentación pelágica del presente y del pasado. Rev. Museo La Plata, N. S., 4, 125-178.

HoEdemarker, P. J.

1973. Olithostromes and other delapsional deposits, and their occurrence in the region of Moratalla (provincia of Murcia, Spain). Scripta geol., 19, 207 páginas.

JENKYNS, H. C.

1971. The genesis of condensed sequences in the Thethyan Jurassic. Lethaia, 4, 327-352.

KRYSTYN, L.; SchÄFFER, G. Y SCHLAGER, W.

1971. Uber die Fossil-Lagerstätten in den triadischen Hallstätter Kalken der Ostalpen. N. Jb. Geol. Paläont. Abh., 137, 284-304.

LAWRENCE, D. R.

1968. Taphonomy and information losses in fossil communities. Geol. Soc. Amer. Bull., 79, 1315-1330.

Margalef, $\mathbf{R}$.

1981. Asimetrías introducidas por la operación de la ener- gía externa en secuencias de sedimentos y de poblaciones. Acta Geológica Hispánica, 16, 1-2 (Cencept and Method in Paleontology, Ed. J. MARTINELL), 35-38, Barcelona.

Matissofr, $\mathbf{G}$.

1982. Mathematical Models of Bioturbation. En: McAlL, P. L. y Tevesz, M. J. S. (Eds.). AnimalSediment Relations. The Biogenic Alteration of sediments, 289-330 (Plenum Press), New YorkLondon.

Mensink, $\mathbf{H}$

1960. Beispiele für die stratigraphische Kondensation Schichtlücke und Leitwert von Ammonites aus dem Jura Spaniens im Vergleich zu NW-Europa. Geol. Rundschau, 49, 70-82.

Müller, A. H

1979. Fossilization (Taphonomy). En: Robinson, R. A. y TeIChert, C. (Eds.). Treatise on Invertebrate Paleontology. Part. A. Introduction, A2-A78 (Geol. Soc. Am. y Univ. Kansas). Boulder, Colorado y Lawrence, Kansas.

OLson, E. C.

1980. Taphonomy: Its History and Role in Community Evolution. En: BeHRENSMEYER, A. K. e HiLl, A. P. (Eds.). Fossils in the Making. Vertebrate Taphonomy and Paleoecology, 5-19 (Univ. Chicago Press), Chicago-London.

Prosser, C. D.

1964. Perspectives of adaptation: theoretical aspects. En: Dill, D. B. (Ed.). Adaptation to the Environment, 11-25 (Amer. Physiol. Soc.), Washington.

QUENSTEDT, W.

1927. Beiträge zum kapitel Fossil und Sediment vor und bei der Einbettung. $N$. Jb. Minenal., Geol., $\mathrm{Pa}$ läont., 58B, 353-432.

RAT, P.

1984. Essai sur la notion de système bio-sédimentaire. Mém. Géol. Univ. Dijon, Liv. Jub. G. Lucas, 7 (1982), 491-591.

RoD, E.

1946. Uber ein Fossillager im oberen Malm der Melchtaleralpen. Eclog. Geol. Helv., 39, 177-198.

SChaUb, H. P.

1948. Über Aufarbeitung und Kondensation. Eclog. Geol. Helv., 41, 84-94.

SeIlacher, A.

1970. Begriff und Bedeutung der Fossil-Lagersttätten. $N$. Jb. Geol Paläont. Mh., 1970, 34-39.

1971. Preservational history of ceratite shells. Palaeontology, 14, 16-21.

SEYFRIED, $\mathbf{H}$.

1981. Genesis of "regressive" and "transgressive" pelagic sequences in the Tethyan Jurassic. En: FARINACCI, A. y ElmI, S. (Eds.). Rosso Ammonitico Symp. Procc., 547-579 (Tecnoscienza). Roma.

ShaPere, D.

1974. Sobre las relaciones entre las teorías composiciones y evolucionistas. En Ayala, F. J. y DoBzHANSKY, T. (Eds.). Estudios sobre la filosofía de la Biología, 246-266 (trad. Ed. Ariel, 1983, Barcelona).

Shipmann, $\mathbf{P}$.

1981. Life History of a Fossil. An Introduction to Ta- 


\title{
NUEVAS PERSPECTIVAS DE LA TAFONOMIA EVOLUTIVA: TAFOSISTEMAS Y ASOCIACIONES CONSERVADAS
}

\author{
S. Fernández López [*]
}

\begin{abstract}
RESUMEN
Varios conceptos básicos de la Tafonomía evolutiva son tratados en este artículo. Hay una discusión general de los procesos principales que controlan la conservación tafonómica. Diversos enfoques para la aplicación de la teoría de la comunicación al registro fósil han sido revisados y sintetizados. Los problemas de resedimentación versus retrabajamiento reciben especial atención como fenómenos tafonómicos. Se hacen algunas observaciones sobre el concepto de condensación.
\end{abstract}

Palabras clave: Paleontología. Paleoecología. Tafonomía. Fosilización. Teoría evolutiva.

\section{Abstract}

Several basic concepts of the evolutionary Taphonomy are examinated in this paper. There is a general discussion of the main processes controlling fossil preservation. Approaches for the aplication of communications theory to the fossil record are reviewed and summarized. The problems of redeposition (Umlagerung) vs reworking (Aufarbeitung) receives special attention as taphonomical phenomena. Some remarks on the concept of condensation are made.

KEY wORDs: Paleontology. Paleoecology. Taphonomy. Fossilization. Evolutionary theory.

\section{Introducción}

En este artículo exponemos los conceptos de asociación conservada y de sistema tafonómico, o tafosistema, además de otros conceptos directamente relacionados con ellos y el de evolución tafonómica, con el propósito de ofrecer una visión sintética de la tafonomía evolutiva. Por este motivo nos ocuparemos de los mecanismos y procesos tafonómicos principales, más que de las diferentes clases de resultados posibles. El reconocimiento o la interpretación de procesos tafonómicos evolutivos, o de entidades conservadas de niveles de organización supraindividuales, no ha de ser necesariamente factible o deseable $y$, en algunos casos concretos, puede estar justificada la utilización de planteamientos individualistas y globalistas. Sin embargo, en el estado actual de los conocimientos, es preferible utilizar un planteamiento sistemista, por razones lógicas y metodológicas, y evolucionista, por su relevancia térica en Paleontología. Naturalmente, cualquier "-ismo" es indeseable.

\section{Acumulación de restos $y / 0$ señales producidas por entidades biológicas}

Desde hace unas décadas, varios autores han utilizado conceptos y términos de la teoría de la información para resolver problemas paleontológicos (cfr. Tasch, 1965, 1969; Beerbower \& Jordan, 1969). Paradójicamente, este enfoque, más que servir para plantear y resolver mejor problemas nuevos y antiguos, parece ser que sólo permite poner de manifiesto la ambigiuedad e imprecisión de algunos conceptos y métodos particulares de la Paleontología. Así, por ejemplo, el problema inicialmente planteado como "la transformación de los restos orgánicos componentes de la Biosfera en componentes de la Litosfera" (Efremov, 1950) ha sido convertido en el de la "transmisión de la información desdo la Biosfera hasta la Litosfera"; pero los conceptos de "registro fósil" y "asociación fósil" siguen siendo demasiado imprecisos para muchos autores; y los procesos de desenterramiento de restos $\mathrm{y} / \mathrm{o}$ señales de entidades bioló gicas históricas son incomprensiblemente tratados como procesos bioestratinómicos.

Diferentes cuestiones relacionadas con el carácter "sedimentario" de los fósiles han sido tratadas en numerosos trabajos. Por ejemplo, se ha dicho que "a geologist who calls a coral reef esedimentary» is extending the normal idea of sediments for sake of making his classification comprehensive and that is in his own interest to be aware of what he is doing. But coral reefs can also be considered as constituted of solid material which has come to rest upon the solid earth's surface by withdrawal of calcium carbonate from the sea water by biochemical processes. In

(*) Departamento de Paleontología de la Universidad Complutense de Madrid e Instituto de Geología Económica del C.S.LC. 
phonomy and Paleoecology, 222 págs. (Harvard Univ. Press), Cambridge-Massachusetts-London.

\section{Spalleta, C.; Perri, M. C. y Vai, G. B.}

1983. Pattern of conodont reworking in the Upper Devonian of the Alpes: palaeoenvironmental and palaeotectonic implications. Lethaia, 16, 51-66.

TASCH, P.

1965. Communications Theory and the Fossil Record of Invertebrates. Trans. Kansas Acad. Sci., 68, 322 . 329.

1969. Diversity Index and Information Concepts. Trans. Kansas Acad. Sci., 72, 192-194.

1969. Information Theory Applied to Invertebrate Fossils. Trans. Kansas Acad. Sci., 72, 195-201.

1982. Experimental Valve Geothermometry Applied to Fossil Conchostracan Valves, Blizard Heigth, Antartica. En: Cradpock, C. (Ed.). Antartic Geoscience. Part. V, Paleontology, 661-668 (Wisconsin Press), Madison.
Tintant, $\mathbf{H}$.

1984. Autochtonie et Allochtonie chez les Céphalopodes. Mém. Géol. Univ. Dijon, Liv. Jub. G. LucAs, 7 (1982), 257-271.

TURNeR, R. E.

1982. Reworked acritarchs from the type section of the Ordovician Caradoc Series, Shropshire. Palaeontology, 25, 119-143.

WASMUND, E.

1926. Biocoenose und Thanatocoenose. Biosoziologische Studie über Lebensgemeinschaften und Totengesellschaften. Arch. Hydrobiol., 17, 1-116.

WENDT, J.

1970. Stratigraphische Kondensation in triadischen und jurassichen Cephalopodenkalken der Tethys. $N$. Jb. Geol. Paläont. Mh., 1970, 433-448.

1973. Cepholopod accumulations in the Middle Triassic Hallstatt-Limestone of Jugoslavia and Grece. $N$. Jb. Geol. Paläont. Mh., 1973, 10, 624-640.

Recibido el 13 de abril de 1984. Aceptado el 2 de julio de 1984.

\section{ADDENDA}

Después de aceptado el manuscrito, los problemas lingüísticos que plantea el término retrabajamiento han sido debatidos por la Comisión de Léxico de la Real Academia de Ciencias Exactas, Físicas y Naturales. Habiéndose aprobado el término reelaboración como sustituto de "retrabajamiento" y con significado tafonómico equivalente al de "reworking" (inglés) y "Aufarbeitung" (alemán), para el que se ha aceptado la siguiente definición: "proceso de alteración tafonómica que consiste en el desenterramiento, desplazamiento y alteración consiguiente, de restos de entidades biológicas pretéritas".

Mi agradecimiento al profesor don Bermudo Meléndez por haber planteado y resuelto este problema linguístico ante dicha comisión. 